Kragujevac Journal of Mathematics

Volume 42(1) (2018), Pages 131-152.

\title{
SOME REFINEMENTS OF CERTAIN GAMIDOV INTEGRAL INEQUALITIES ON TIME SCALES AND APPLICATIONS
}

\author{
K. BOUKERRIOUA ${ }^{1}$, I. MEZIRI ${ }^{1}$, AND T. CHIHEB $^{2}$
}

\begin{abstract}
The goal of this paper is to derive some generalizations and refinements of certain Gamidov type integral inequalities on time scales, which provide explicit bounds on unknown functions. To show the feasibility of the obtained inequalities, some illustrative examples are also introduced.
\end{abstract}

\section{INTRODUCTION}

Integral inequalities that give explicit bounds on unknown functions provide a very useful and important device in the study of many qualitative as well as quantitative properties of solutions of differential and integral equations. During the past few years, many such new inequalities have been discovered, which are motivated by certain applications. For example, see [2-4,7-20] and the references therein. In [14], Sh. Gamidov, while studying the boundary value problem for higher order differential equations, initiated the study of obtaining explicit upper bounds on the integral inequalities of the forms

$$
u(t) \leq c+\int_{a}^{t} a(s) u(s) d s+\int_{a}^{b} b(s) u(s) d s .
$$

In [19], Pachpatte established more general Gamidov inequalities as

$$
u(t) \leq a(t)+\int_{a}^{t} b(t, s) u(s) d s+\int_{a}^{b} c(s) u(s) d s .
$$

Key words and phrases. Dynamic equations, time scale, Gamidov integral inequality. 2010 Mathematics Subject Classification. Primary: 26D15. Secondary: 26D20, 39A12. Received: April 9, 2016.

Accepted: December 12, 2016. 
In the present paper we shall consider the problem of obtaining explicit upper bounds on the general versions of (1.1) on time scales which can be used as tools in the study of qualitative behaviour of solutions of certain classes of integral equations on time scales.

\section{Some PRELIMINARIES}

In what follows, $\mathbb{R}$ denotes the set of real numbers, $\mathbb{R}_{+}=[0, \infty)$ is the given subset of $\mathbb{R}$ and $\mathbb{T}$ is an arbitrary time scale. The forward and backward jump operators $\sigma, \rho: \mathbb{T} \rightarrow \mathbb{T}$ are defined by $\sigma(t):=\inf \{s \in \mathbb{T}: s>t\}, \rho(t):=\sup \{s \in \mathbb{T}: s<t\}$. $C_{r d}$ denotes the set of rd-continuous functions and the set $\mathbb{T}^{k}$ which is derived from the time scale $\mathbb{T}$ as follows: If $\mathbb{T}$ has a left-scattered maximum $m$, then $\mathbb{T}^{k}=\mathbb{T}-\{m\}$, otherwise, $\mathbb{T}^{k}=\mathbb{T}$. The graininess function $\mu: \mathbb{T} \rightarrow[0, \infty[$ is defined by $\mu(t):=\sigma(t)-t$. $\mathfrak{R}$ denotes the set of all regressive and $r d$-continuous functions.

We define the set of all positively regressive functions by

$$
\mathfrak{R}^{+}=\{p \in \mathfrak{R}: 1+\mu(t) p(t)>0, \text { for all } t \in \mathbb{T}\} .
$$

Also, we define the time scales interval by

$$
[a, b]_{\mathbb{T}}=\{t \in \mathbb{T}: a \leq t \leq b\},
$$

note that $[a, b]_{\mathbb{T}}^{k}= \begin{cases}{[a, b]_{\mathbb{T}},} & \text { if } b \text { is left- dense, } \\ {[a, b]_{\mathbb{T}}=[a, \rho(b)]_{\mathbb{T}}=[a, b[,} & \text { if } b \text { is left- scattered. }\end{cases}$

Definition 2.1. If $p \in \mathfrak{R}(\mathbb{T}, \mathbb{R})$, then we define the generalized exponential function $e_{p}(t, s)$ by

$$
e_{p}(t, s)=\exp \left(\int_{s}^{t} \xi_{\mu(\tau)}(p(\tau)) \Delta \tau\right), \quad \text { for } s, t \in \mathbb{T},
$$

where $\xi_{h}(z)$ is the cylinder transformation given by

$$
\begin{aligned}
& \xi_{h}(z)=\frac{1}{h} \log (1+z h), \quad \text { if } h \neq 0, \\
& \xi_{0}(z)=z, \quad \text { if } h=0 .
\end{aligned}
$$

Here log is the principal logarithm function.

The following lemmas are useful in our main results.

Lemma 2.1. [5, Theorem 6.1] Suppose $u, b \in C_{r d}, a \in \mathfrak{R}^{+}$. Then

$$
u^{\Delta}(t) \leq a(t) u(t)+b(t), \quad t \geq t_{0}, t \in \mathbb{T},
$$

implies

$$
u(t) \leq u\left(t_{0}\right) e_{a}\left(t, t_{0}\right)+\int_{t_{0}}^{t} b(\tau) e_{a}(t, \sigma(\tau)) \Delta \tau, \quad t \geq t_{0}, t \in \mathbb{T}
$$


Lemma 2.2. [5, Theorem 1.117] Let $a \in \mathbb{T}^{k}, b \in \mathbb{T}$ and assume $f: \mathbb{T} \times \mathbb{T}^{k} \rightarrow \mathbb{R}$ is continuous at $(t, t)$, where $t \in \mathbb{T}^{k}$ with $t>a$. Also assume that $f^{\Delta}(t,$.$) is r d$ continuous on $[a, \sigma(t)]$. Suppose that for each $\varepsilon>0$ there exists a neighborhood $U$ of $t$, independent of $\tau \in[a, \sigma(t)]$, such that

$$
\left|f(\sigma(t), \tau)-f(s, \tau)-f^{\Delta}(t, \tau)(\sigma(t)-s)\right| \leq \varepsilon|\sigma(t)-s|, \quad \text { for all } s \in U,
$$

where $f^{\Delta}$ denotes the derivative of $f$ with respect to the first variable. Then

$$
g(t):=\int_{a}^{t} f(t, \tau) \Delta \tau
$$

implies

$$
g^{\Delta}(t)=\int_{a}^{t} f^{\Delta}(t, \tau) \Delta \tau+f(\sigma(t), t) .
$$

For more discussion on time scales, we refer the reader to $[1,5,6]$.

Lemma 2.3. [13, Lemma 2.1] Let $a, b \in \mathbb{T}$, consider the time scales interval $[a, b]_{\mathbb{T}}$ and a delta differentiable function $\left.r:[a, b]_{\mathbb{T}} \rightarrow\right] 0, \infty\left[\right.$ with $r^{\Delta} \geq 0$ on $[a, b]_{\mathbb{T}}^{k}$. Define

$$
N(x)=\int_{x_{0}}^{x} \frac{d s}{n(s)}, \quad x>x_{0}>0,
$$

where $n \in C\left(\mathbb{R}_{+}, \mathbb{R}_{+}\right)$is positive and non-decreasing on $] 0, \infty[$. Then, for each $t \in$ $[a, b]_{\mathbb{T}}$, we have

$$
N(r(t)) \leq N(r(a))+\int_{a}^{t} \frac{r^{\Delta}(\tau)}{n(r(\tau))} \Delta \tau .
$$

Lemma 2.4. [15, Lemma 2.1] Assume that $a \geq 0, p \geq q>0$, then

$$
a^{\frac{q}{p}} \leq \frac{q}{p} K^{\frac{q-p}{p}} a+\frac{p-q}{p} K^{\frac{q}{p}}
$$

for any $K>0$.

Definition 2.2. A non-decreasing, continuous function $\eta: \mathbb{R}_{+} \rightarrow \mathbb{R}_{+}$is said to belong to class $\mathcal{S}$, if it satisfies the following conditions

$$
\begin{aligned}
\eta(x) & >0, \quad \text { for } x>0, \\
\frac{1}{a} \eta(x) & \leq \eta\left(\frac{x}{a}\right), \quad \text { for } x \geq 0 \text { and } a \geq 1 .
\end{aligned}
$$


Definition 2.3. A non-decreasing, continuous function $\eta: \mathbb{R}_{+} \rightarrow \mathbb{R}_{+}$is said to belong to class $\mathcal{T}$, if it satisfies the following conditions

$$
\begin{aligned}
\eta(x) & >0, \quad \text { for } \quad x>0, \\
\frac{1}{a} \eta(x) & \geq \eta\left(\frac{x}{a}\right), \quad \text { for all } x \geq 0 \text { and } a \geq 1 .
\end{aligned}
$$

Now we state the main results of this work.

\section{Main Results}

In this section, some time scale Gamidov type integral inequalities are investigated. For convenience, it is always assumed that $p \neq 0, p, q, r$ are real constants such that $0 \leq q, r \leq p$ and $a, b \in \mathbb{T}$.

Lemma 3.1. Suppose $u, m, l$ and $n \in C_{r d}\left([a, b]_{\mathbb{T}}, \mathbb{R}_{+}\right)$. If

$$
u(t) \leq m(t)+l(t) \int_{a}^{b} n(s) u(s) \Delta s
$$

then

$$
u(t) \leq m(t)+\frac{l(t) \int_{a}^{b} n(s) m(s) \Delta s}{1-\int_{a}^{b} n(s) l(s) \Delta s},
$$

for $t \in[a, b]_{\mathbb{T}}^{k}$, provided that

$$
\int_{a}^{b} n(s) l(s) \Delta s<1
$$

Proof. Let

$$
k=\int_{a}^{b} n(s) u(s) \Delta s .
$$

Obviously that $k$ is a constant. It follows from (3.1) that

$$
u(t) \leq m(t)+l(t) k .
$$

Multiplying both sides of (3.4) by $n(t)$, then integrating the result from $a$ to $b$, we have

It is easy to observe that

$$
\int_{a}^{b} n(s) u(s) \Delta s \leq \int_{a}^{b} n(s) m(s) \Delta s+k \int_{a}^{b} n(s) l(s) \Delta s .
$$

$$
k \leq \int_{a}^{b} n(s) m(s) \Delta s+k \int_{a}^{b} n(s) l(s) \Delta s
$$

the inequality (3.5) implies the estimate

$$
\left(1-\int_{a}^{b} n(s) l(s) \Delta s\right) k \leq \int_{a}^{b} n(s) m(s) \Delta s
$$


from (3.3), we observe that

$$
k \leq \frac{\int_{a}^{b} n(s) m(s) \Delta s}{1-\int_{a}^{b} n(s) l(s) \Delta s} .
$$

Therefore, the desired inequality (3.2) follows from (3.6) and (3.4).

Theorem 3.1. Assume that $u, c, g \in C_{r d}\left([a, b]_{\mathbb{T}}, \mathbb{R}_{+}\right)$and $c^{\Delta} \geq 0$. If $f$ is defined as in Lemma 2.2 such that $f(t, s) \geq 0$ and $f^{\Delta}(t, s) \geq 0$ for $t, s \in[a, b]_{\mathbb{T}}$ with $s \leq t$. Then

$$
u^{p}(t) \leq c(t)+\int_{a}^{t} f(t, s) u^{q}(s) \Delta s+\int_{a}^{b} g(s) u^{r}(s) \Delta s
$$

implies

$$
u(t) \leq\left[m(t)+\frac{\frac{r}{p} k^{\frac{r-p}{p}} e_{P}(t, a) \int_{a}^{b} g(s) m(s) \Delta s}{1-\frac{r}{p} k^{\frac{r-p}{p}} \int_{a}^{b} g(s) e_{P}(s, a) \Delta s}\right]^{\frac{1}{p}},
$$

for $t \in[a, b]_{\mathbb{T}}^{k}$, where

$$
\begin{aligned}
& P(t)=\frac{q}{p} k^{\frac{q-p}{p}}\left[f(\sigma(t), t)+\int_{a}^{t} f^{\Delta}(t, s) \Delta s\right], \\
& Q(t)=c^{\Delta}(t)+\frac{p-q}{p} k^{\frac{q}{p}}\left[f(\sigma(t), t)+\int_{a}^{t} f^{\Delta}(t, s) \Delta s\right],
\end{aligned}
$$

and

$$
m(t)=c(a) e_{P}(t, a)+\int_{a}^{t} Q(s) e_{P}(t, \sigma(s)) \Delta s+\frac{p-r}{p} k^{\frac{r}{p}} e_{P}(t, a) \int_{a}^{b} g(s) \Delta s
$$

with

$$
\frac{r}{p} k^{\frac{r-p}{p}} \int_{a}^{b} g(t) e_{P}(t, a) \Delta t<1
$$

Proof. Define a function $z(t)$ by

$$
z(t)=c(t)+\int_{a}^{t} f(t, s) u^{q}(s) \Delta s+\int_{a}^{b} g(s) u^{r}(s) \Delta s
$$

then

$$
\begin{aligned}
u(t) & \leq z^{\frac{1}{p}}(t) \\
z(a) & =c(a)+\int_{a}^{b} g(s) u^{r}(s) \Delta s \leq c(a)+\int_{a}^{b} g(s) z^{\frac{r}{p}}(s) \Delta s .
\end{aligned}
$$

And

$$
\begin{aligned}
z^{\Delta}(t) & =c^{\Delta}(t)+f(\sigma(t), t) u^{q}(t)+\int_{a}^{t} f^{\Delta}(t, s) u^{q}(s) \Delta s \\
& \leq c^{\Delta}(t)+\left[f(\sigma(t), t)+\int_{a}^{t} f^{\Delta}(t, s) \Delta s\right] z^{\frac{q}{p}}(t)
\end{aligned}
$$


Using Lemma 2.4, for any $k>0$, we easily obtain

$$
z^{\Delta}(t) \leq c^{\Delta}(t)+\left(f(\sigma(t), t)+\int_{a}^{t} f^{\Delta}(t, s) \Delta s\right)\left(\frac{q}{p} K^{\frac{q-p}{p}} z(t)+\frac{p-q}{p} K^{\frac{q}{p}}\right),
$$

the above inequality can be reformulated as

$$
z^{\Delta}(t) \leq P(t) z(t)+Q(t), \quad t \in[a, b]_{\mathbb{T}}^{k},
$$

where $P$ and $Q$ are defined as in (3.8).

Using Lemma 2.1 from (3.11), we have

$$
z(t) \leq z(a) e_{P}(t, a)+\int_{a}^{t} Q(s) e_{P}(t, \sigma(s)) \Delta s, t \in[a, b]_{\mathbb{T}}^{k} .
$$

Combining the above inequality with (3.10), one can obtain

$$
z(t) \leq c(a) e_{P}(t, a)+\int_{a}^{t} Q(s) e_{P}(t, \sigma(s)) \Delta s+e_{P}(t, a) \int_{a}^{b} g(s) z^{\frac{r}{p}}(s) \Delta s .
$$

Using Lemma 2.4 from (3.12), we obtain

$$
\begin{aligned}
z(t) \leq & c(a) e_{P}(t, a)+\int_{a}^{t} Q(s) e_{P}(t, \sigma(s)) \Delta s \\
& +e_{P}(t, a) \int_{a}^{b} g(s)\left(\frac{r}{p} K^{\frac{r-p}{p}} z(s)+\frac{p-r}{p} K^{\frac{r}{p}}\right) \Delta s
\end{aligned}
$$

the above inequality can be reformulated as

$$
z(t) \leq m(t)+\frac{r}{p} k^{\frac{r-p}{p}} e_{P}(t, a) \int_{a}^{b} g(s) z(s) \Delta s, \quad t \in[a, b]_{\mathbb{T}}^{k},
$$

where $m$ is defined as in (3.9).

Applying Lemma 3.1 and using (3.10), we obtain the desired inequality (3.7).

Remark 3.1. If we take $\mathbb{T}=\mathbb{R}, p=q=r=1$, the inequality given in Theorem 3.1 reduces to the inequality given in $\left[19\right.$, Theorem $\left.1,\left(a_{3}\right)\right]$.

Theorem 3.2. Assume that $u, h, g \in C_{r d}\left([a, b]_{\mathbb{T}}, \mathbb{R}_{+}\right)$and $c \geq 0$ be a constant. If $f$ is defined as in Lemma 2.2 such that $f(t, s) \geq 0$ and $f^{\Delta}(t, s) \geq 0$ for $t, s \in[a, b]_{\mathbb{T}}$ with $s \leq t$. Then

$$
u^{p}(t) \leq c+\int_{a}^{t} h(s)\left[u^{q}(s)+\int_{a}^{s} f(s, \tau) u^{q}(\tau) \Delta \tau+\int_{a}^{b} g(\tau) u^{r}(\tau) \Delta \tau\right] \Delta s,
$$

implies

$$
u(t) \leq\left[c+\int_{a}^{t} h(s)\left(m(s)+\frac{l(s) \int_{a}^{b} g(\tau) m(\tau) \Delta \tau}{1-\int_{a}^{b} g(\tau) l(\tau) \Delta \tau}\right) \Delta s\right]^{\frac{1}{p}}
$$


for $t \in[a, b]_{\mathbb{T}}^{k}$, provided that

$$
\int_{a}^{b} g(\tau) l(\tau) \Delta \tau<1
$$

where

$$
\begin{aligned}
m(t)= & \left(\frac{q}{p} K^{\frac{q-p}{p}} c+\frac{p-q}{p} K^{\frac{q}{p}}+\frac{p-r}{p} K^{\frac{r}{p}} \int_{a}^{b} g(\tau) \Delta \tau\right) e_{P}(t, a) \\
& +\int_{a}^{t} Q(\tau) e_{P}(t, \sigma(\tau)) \Delta \tau
\end{aligned}
$$

and

$$
\begin{aligned}
& P(t)=\frac{q}{p} K^{\frac{q-p}{p}} h(t)+f(\sigma(t), t)+\int_{a}^{t} f^{\Delta}(t, \tau) \Delta \tau, \\
& Q(t)=\frac{p-q}{p} K^{\frac{q}{p}}\left(f(\sigma(t), t)+\int_{a}^{t} f^{\Delta}(t, \tau) \Delta \tau\right) .
\end{aligned}
$$

Proof. Define a function $z(t)$ as follows

$$
z(t)=c+\int_{\alpha}^{t} h(s)\left[u^{q}(s)+\int_{a}^{s} f(s, \tau) u^{q}(\tau) \Delta \tau+\int_{a}^{b} g(\tau) u^{r}(\tau) \Delta \tau\right] \Delta s,
$$

then

$$
\begin{gathered}
z(a)=c, \\
u(t) \leq z^{\frac{1}{p}}(t),
\end{gathered}
$$

and

$$
z^{\Delta}(t)=h(t)\left[u^{q}(t)+\int_{a}^{t} f(t, \tau) u^{q}(\tau) \Delta \tau+\int_{a}^{b} g(\tau) u^{r}(\tau) \Delta \tau\right] .
$$

Using (3.16) in (3.17), we get

$$
z^{\Delta}(t) \leq h(t)\left[\left(z^{\frac{q}{p}}(t)+\int_{a}^{t} f(t, \tau) z^{\frac{q}{p}}(\tau) \Delta \tau\right)+\left(\int_{a}^{b} g(\tau) z^{\frac{r}{p}}(\tau) \Delta \tau\right)\right] .
$$

Using Lemma 2.4 from (3.18), we obtain

$$
\begin{aligned}
z^{\Delta}(t) \leq & h(t)\left[\left(\frac{q}{p} K^{\frac{q-p}{p}} z(t)+\frac{p-q}{p} K^{\frac{q}{p}}+\int_{a}^{t} f(t, \tau)\left(\frac{q}{p} K^{\frac{q-p}{p}} z(\tau)+\frac{p-q}{p} K^{\frac{q}{p}}\right) \Delta \tau\right)\right. \\
& \left.+\left(\int_{a}^{b} g(\tau)\left(\frac{r}{p} K^{\frac{r-p}{p}} z(\tau)+\frac{p-r}{p} K^{\frac{r}{p}}\right) \Delta \tau\right)\right] .
\end{aligned}
$$


Define a function $v(t)$ by

$$
\begin{aligned}
v(t)= & \left(\left(\frac{q}{p} K^{\frac{q-p}{p}} z(t)+\frac{p-q}{p} K^{\frac{q}{p}}\right)+\int_{a}^{t} f(t, \tau)\left(\frac{q}{p} K^{\frac{q-p}{p}} z(\tau)+\frac{p-q}{p} K^{\frac{q}{p}}\right) \Delta \tau\right) \\
& +\left(\int_{a}^{b} g(\tau)\left(\frac{r}{p} K^{\frac{r-p}{p}} z(\tau)+\frac{p-r}{p} K^{\frac{r}{p}}\right) \Delta \tau\right),
\end{aligned}
$$

then

$$
v(a)=\frac{q}{p} K^{\frac{q-p}{p}} c+\frac{p-q}{p} K^{\frac{q}{p}}+\int_{a}^{b} g(\tau)\left(\frac{r}{p} K^{\frac{r-p}{p}} z(\tau)+\frac{p-r}{p} K^{\frac{r}{p}}\right) \Delta \tau .
$$

It is easy to observe that

$$
\frac{q}{p} K^{\frac{q-p}{p}} z(t) \leq v(t), \quad z^{\Delta}(t) \leq h(t) v(t)
$$

and $v(t)$ is non-decreasing for $t \in[a, b]_{\mathbb{T}}^{k}$.

Using Lemma 2.2, we get

$$
\begin{aligned}
v^{\Delta}(t)= & \frac{q}{p} K^{\frac{q-p}{p}} z^{\Delta}(t)+f(\sigma(t), t)\left(\frac{q}{p} K^{\frac{q-p}{p}} z(t)+\frac{p-q}{p} K^{\frac{q}{p}}\right) \\
& +\int_{a}^{t} f^{\Delta}(t, \tau)\left(\frac{q}{p} K^{\frac{q-p}{p}} z(\tau)+\frac{p-q}{p} K^{\frac{q}{p}}\right) \Delta \tau
\end{aligned}
$$

it follows from (3.21) and (3.20) that

$$
\begin{aligned}
v^{\Delta}(t) \leq & \left(\frac{q}{p} K^{\frac{q-p}{p}} h(t)+f(\sigma(t), t)+\int_{a}^{t} f^{\Delta}(t, \tau) \Delta \tau\right) v(t) \\
& +\frac{p-q}{p} K^{\frac{q}{p}}\left(f(\sigma(t), t)+\int_{a}^{t} f^{\Delta}(t, \tau) \Delta \tau\right),
\end{aligned}
$$

then the above inequality can be reformulated as

$$
v^{\Delta}(t) \leq P(t) v(t)+Q(t)
$$

where $P(t)$ and $Q(t)$ are defined as in (3.15).

Using Lemma 2.1 from (3.22), we have

$$
v(t) \leq v(a) e_{P}(t, a)+\int_{a}^{t} Q(\tau) e_{P}(t, \sigma(\tau)) \Delta \tau
$$

From (3.19), we get

$$
\begin{aligned}
v(t) \leq & \left(\frac{q}{p} K^{\frac{q-p}{p}} c+\frac{p-q}{p} K^{\frac{q}{p}}+\int_{a}^{b} g(\tau)\left(\frac{r}{p} K^{\frac{r-p}{p}} z(\tau)+\frac{p-r}{p} K^{\frac{r}{p}}\right) \Delta \tau\right) \\
& \times e_{P}(t, a)+\int_{a}^{t} Q(\tau) e_{P}(t, \sigma(\tau)) \Delta \tau,
\end{aligned}
$$


it follows from (3.20) that

$$
\frac{r}{p} K^{\frac{r-p}{p}} z(t) \leq \frac{\frac{r}{p} K^{\frac{r-p}{p}}}{\frac{q}{p} K^{\frac{q-p}{p}}} \frac{q}{p} K^{\frac{q-p}{p}} z(t) \leq \frac{r}{q} K^{\frac{r-q}{p}} v(t),
$$

therefore

$$
\begin{aligned}
v(t) \leq & \left(\frac{q}{p} K^{\frac{q-p}{p}} c+\frac{p-q}{p} K^{\frac{q}{p}}+\frac{p-r}{p} K^{\frac{r}{p}} \int_{a}^{b} g(\tau) \Delta \tau\right) e_{P}(t, a) \\
& +\int_{a}^{t} Q(\tau) e_{P}(t, \sigma(\tau)) \Delta \tau+\frac{r}{q} K^{\frac{r-q}{p}} e_{P}(t, a) \int_{a}^{b} g(\tau) v(\tau) \Delta \tau
\end{aligned}
$$

the above inequality can be reformulated as

$$
v(t) \leq m(t)+l(t) \int_{a}^{b} g(\tau) v(\tau) \Delta \tau
$$

with

$$
l(t)=\frac{r}{q} K^{\frac{r-q}{p}} e_{P}(t, a),
$$

and $m(t)$ is defined as in (3.14).

Applying Lemma 3.1 to (3.23) we obtain

$$
v(t) \leq m(t)+\frac{l(t) \int_{a}^{b} g(\tau) m(\tau) \Delta \tau}{1-\int_{a}^{b} g(\tau) l(\tau) \Delta \tau}
$$

Thus, from (3.20), one gets

$$
z^{\Delta}(t) \leq h(t)\left(m(t)+\frac{l(t) \int_{a}^{b} g(\tau) m(\tau) \Delta \tau}{1-\int_{a}^{b} g(\tau) l(\tau) \Delta \tau}\right) .
$$

Integrating both sides of the last inequality from $a$ to $t$, yields

$$
z(t) \leq c+\int_{a}^{t} h(s)\left(m(s)+\frac{l(s) \int_{a}^{b} g(\tau) m(\tau) \Delta \tau}{1-\int_{a}^{b} g(\tau) l(\tau) \Delta \tau}\right) \Delta s .
$$

Therefore, the desired inequality (3.13) follows from (3.24) and (3.16).

Remark 3.2. Note that when $\mathbb{T}=\mathbb{R}, r=p, f(t, s)=f(t)$, the Theorem 3.2 reduces to the inequality stated in Theorem 3.2 in [16].

Corollary 3.1. Assume that all assumptions of Theorem 3.2 hold. Let $1 \leq c(t)$ be a non-decreasing function. Then

$$
u^{p}(t) \leq c^{p}(t)+\int_{a}^{t} h(s)\left[u^{q}(s)+\int_{a}^{s} f(s, \tau) u^{q}(\tau) \Delta \tau+\int_{a}^{b} g(\tau) u^{r}(\tau) \Delta \tau\right] \Delta s
$$


implies

$$
u(t) \leq c(t)\left[1+\int_{a}^{t} h(s)\left(m(s)+\frac{l(s) \int_{a}^{b} g(\tau) m(\tau) \Delta \tau}{1-\int_{a}^{b} g(\tau) l(\tau) \Delta \tau}\right) \Delta s\right]^{\frac{1}{p}}
$$

for $t \in[a, b]_{\mathbb{T}}^{k}$,

$$
\begin{aligned}
m(t)= & \left(\frac{q}{p} K^{\frac{q-p}{p}}+\frac{p-q}{p} K^{\frac{q}{p}}+\frac{p-r}{p} K^{\frac{r}{p}} \int_{a}^{b} g(\tau) \Delta \tau\right) e_{P}(t, a) \\
& +\int_{a}^{t} Q(\tau) e_{P}(t, \sigma(\tau)) \Delta \tau, \\
l(t)= & \frac{r}{q} K^{\frac{r-q}{p}} e_{P}(t, a),
\end{aligned}
$$

and

$$
\begin{aligned}
& P(t)=\frac{q}{p} K^{\frac{q-p}{p}} h(t)+f(\sigma(t), t)+\int_{a}^{t} f^{\Delta}(t, \tau) \Delta \tau \\
& Q(t)=\frac{p-q}{p} K^{\frac{q}{p}}\left(f(\sigma(t), t)+\int_{a}^{t} f^{\Delta}(t, \tau) \Delta \tau\right),
\end{aligned}
$$

with

$$
\int_{a}^{b} g(\tau) l(\tau) \Delta \tau<1
$$

Proof. Since $1 \leq c(t)$ and non-decreasing, one can reformulate (3.25) as

$$
\begin{aligned}
w^{p}(t) & =\left(\frac{u(t)}{c(t)}\right)^{p} \\
& \leq 1+\int_{a}^{t} h(s)\left[w^{q}(s)+\int_{a}^{s} f(s, \tau) w^{q}(\tau) \Delta \tau+\int_{a}^{b} g(\tau) w^{r}(\tau) \Delta \tau\right] \Delta s
\end{aligned}
$$

applying Theorem 3.2 to (3.27), one can deduce inequality (3.26).

Remark 3.3. It is interesting to note that when $\mathbb{T}=\mathbb{R}, r=p, f(t, s)=f(t)$, the Corollary 3.1 reduces to the inequality stated in Corollary 3.3 in [16].

\section{Further Results}

In this section we present some extensions of the previous results.

Theorem 4.1. Assume that all assumptions of Theorem 3.1 hold. Let $S:[a, b]_{\mathbb{T}}^{k} \times$ $\mathbb{R}_{+} \rightarrow \mathbb{R}_{+}$be an rd-continuous function satisfying

$$
\begin{aligned}
0 & \leq S(t, x)-S(t, y) \leq R(t, y)(x-y), \\
S^{\Delta}(t, 0) & \geq 0, R^{\Delta}(t, 0) \geq 0,
\end{aligned}
$$


for $t \in[a, b]_{\mathbb{T}}^{k}$ and $x \geq y \geq 0$, where $R:[a, b]_{\mathbb{T}}^{k} \times \mathbb{R}_{+} \rightarrow \mathbb{R}_{+}^{*}$ is an rd-continuous function. Then

$$
u^{p}(t) \leq c(t)+\int_{a}^{t} f(t, \tau) S\left(\tau, u^{q}(\tau)\right) \Delta \tau+\int_{a}^{b} g(\tau) S\left(\tau, u^{r}(\tau)\right) \Delta \tau
$$

implies

$$
u(t) \leq\left\{m(t)+\frac{l(t) \int_{a}^{b} g(\tau) R\left(\tau, \frac{p-r}{p} K^{\frac{r}{p}}\right) m(\tau) \Delta \tau}{1-\int_{a}^{b} g(\tau) R\left(\tau, \frac{p-r}{p} K^{\frac{r}{p}}\right) l(\tau) \Delta \tau}\right\}^{\frac{1}{p}}
$$

for $t \in[a, b]_{\mathbb{T}}^{k}$, where

$$
\begin{aligned}
l(t)= & \frac{r}{p} K^{\frac{r-p}{p}} e_{P}(t, a) \\
m(t)= & \left(c(a)+\int_{a}^{b} g(\tau) S\left(\tau, \frac{p-r}{p} K^{\frac{r}{p}}\right) \Delta \tau\right) e_{P}(t, a) \\
& +\int_{a}^{t} Q(\tau) e_{P}(t, \sigma(\tau)) \Delta \tau
\end{aligned}
$$

and

$$
\begin{aligned}
P(t)= & \frac{q}{p} K^{\frac{q-p}{p}} R\left(t, \frac{p-q}{p} K^{\frac{q}{p}}\right) f(\sigma(t), t) \\
& +\int_{a}^{t} \frac{q}{p} K^{\frac{q-p}{p}} R\left(\tau, \frac{p-q}{p} K^{\frac{q}{p}}\right) f^{\Delta}(t, \tau) \Delta \tau \\
Q(t)= & c^{\Delta}(t)+f(\sigma(t), t) S\left(t, \frac{p-q}{p} K^{\frac{q}{p}}\right)+\int_{a}^{t} f^{\Delta}(t, \tau) S\left(\tau, \frac{p-q}{p} K^{\frac{q}{p}}\right) \Delta \tau .
\end{aligned}
$$

Proof. Define a function $z(t)$ as follows

$$
z(t)=c(t)+\int_{a}^{t} f(t, \tau) S\left(\tau, u^{q}(\tau)\right) \Delta \tau+\int_{a}^{b} g(\tau) S\left(\tau, u^{r}(\tau)\right) \Delta \tau
$$

then

$$
z(a)=c(a)+\int_{a}^{b} g(\tau) S\left(\tau, u^{r}(\tau)\right) \Delta \tau
$$

and

$$
u(t) \leq z^{\frac{1}{p}}(t) .
$$

From Lemma 2.4 and using the first condition of (4.1), one has for any $K>0$

$$
\begin{aligned}
S\left(t, u^{r}(t)\right) & \leq S\left(t, z^{\frac{r}{p}}(t)\right) \leq S\left(t, \frac{r}{p} K^{\frac{r-p}{p}} z(t)+\frac{p-r}{p} K^{\frac{r}{p}}\right) \\
& \leq \frac{r}{p} K^{\frac{r-p}{p}} R\left(t, \frac{p-r}{p} K^{\frac{r}{p}}\right) z(t)+S\left(t, \frac{p-r}{p} K^{\frac{r}{p}}\right) .
\end{aligned}
$$


From (4.7) and (4.9), we have

$$
z(a) \leq c(a)+\int_{a}^{b} g(\tau)\left[\frac{r}{p} K^{\frac{r-p}{p}} R\left(\tau, \frac{p-r}{p} K^{\frac{r}{p}}\right) z(\tau)+S\left(\tau, \frac{p-r}{p} K^{\frac{r}{p}}\right)\right] \Delta \tau \text {. }
$$

The $\Delta$-derivation of (4.6), gives

$$
z^{\Delta}(t)=c^{\Delta}(t)+f(\sigma(t), t) S\left(t, u^{q}(t)\right)+\int_{a}^{t} f^{\Delta}(t, \tau) S\left(\tau, u^{q}(\tau)\right) \Delta \tau,
$$

then, from (4.9), we obtain

$$
\begin{aligned}
(4.11) z^{\Delta}(t) \leq & c^{\Delta}(t)+f(\sigma(t), t)\left[\frac{q}{p} K^{\frac{q-p}{p}} R\left(t, \frac{p-q}{p} K^{\frac{q}{p}}\right) z(t)+S\left(t, \frac{p-q}{p} K^{\frac{q}{p}}\right)\right] \\
& +\int_{a}^{t} f^{\Delta}(t, \tau)\left[\frac{q}{p} K^{\frac{q-p}{p}} R\left(\tau, \frac{p-q}{p} K^{\frac{q}{p}}\right) z(\tau)+S\left(\tau, \frac{p-q}{p} K^{\frac{q}{p}}\right)\right] \Delta \tau,
\end{aligned}
$$

the inequality (4.11) can be reformulated as

$$
z^{\Delta}(t) \leq P(t) z(t)+Q(t)
$$

where $P(t)$ and $Q(t)$ are defined as in (4.5).

Applying Lemma 2.1 to (4.12) we obtain

$$
z(t) \leq z(a) e_{P}(t, a)+\int_{a}^{t} Q(\tau) e_{P}(t, \sigma(\tau)) \Delta \tau
$$

using (4.10), we have

$$
\begin{aligned}
z(t) \leq & \left(c(a)+\int_{a}^{b} g(\tau)\left[\frac{r}{p} K^{\frac{r-p}{p}} R\left(\tau, \frac{p-r}{p} K^{\frac{r}{p}}\right) z(\tau)+S\left(\tau, \frac{p-r}{p} K^{\frac{r}{p}}\right)\right] \Delta \tau\right) e_{P}(t, a) \\
& +\int_{a}^{t} Q(\tau) e_{P}(t, \sigma(\tau)) \Delta \tau .
\end{aligned}
$$

The inequality (4.13) can be rewritten as

$$
z(t) \leq m(t)+l(t) \int_{a}^{b} g(\tau) R\left(\tau, \frac{p-r}{p} K^{\frac{r}{p}}\right) z(\tau) \Delta \tau
$$

where $l(t), m(t)$ are defined as in (4.3) and (4.4).

Using Lemma 3.1, inequality (4.14) becomes

$$
z(t) \leq m(t)+\frac{l(t) \int_{a}^{b} g(\tau) R\left(\tau, \frac{p-r}{p} K^{\frac{r}{p}}\right) m(\tau) \Delta \tau}{1-\int_{a}^{b} g(\tau) R\left(\tau, \frac{p-r}{p} K^{\frac{r}{p}}\right) l(\tau) \Delta \tau} .
$$

Combining the above inequality with (4.8), one can deduce inequality (4.2). 
Remark 4.1. Letting $\mathbb{T}=\mathbb{R}, S(t, u(t))=u(t), f(t, s)=f(t), p=q=r=1$ and $c(t)=c$ (a constant), the inequality given in Theorem 4.1 reduces to the inequality given in [18, Lemma BS] and when $\mathbb{T}=\mathbb{R}, S(t, u(t))=u(t), r=p$, and $f(t, s)=f(t)$, Theorem 4.1 will be reduced to Theorem 3.1 in [16].

Theorem 4.2. Assume that all conditions of Theorem 3.2 are satisfied. If $S$ is defined as in Theorem 4.1, then

$u^{p}(t) \leq c+\int_{a}^{t} h(s)\left[S\left(s, u^{q}(s)\right)+\int_{a}^{s} f(s, \tau) S\left(\tau, u^{q}(\tau)\right) \Delta \tau+\int_{a}^{b} g(\tau) S\left(\tau, u^{r}(\tau)\right) \Delta \tau\right] \Delta s$,

implies

$$
u(t) \leq\left\{c+\int_{a}^{t} h(s)\left[m(s)+\frac{l(s) \int_{a}^{b} \frac{R\left(\tau, \frac{p-r}{p} K^{\frac{r}{p}}\right) g(\tau)}{R\left(\tau, \frac{p-q}{p} K^{\frac{q}{p}}\right)} m(\tau) \Delta \tau}{1-\int_{a}^{b} \frac{R\left(\tau, \frac{p-r}{p} K^{\frac{r}{p}}\right) g(\tau)}{R\left(\tau, \frac{p-q}{p} K^{\frac{q}{p}}\right)} l(\tau) \Delta \tau}\right] \Delta s\right\}^{\frac{1}{p}},
$$

for $t \in[a, b]_{\mathbb{T}}^{k}$, where

$$
\begin{aligned}
m(t)= & {\left[\frac{q}{p} K^{\frac{q-p}{p}} R\left(a, \frac{p-q}{p} K^{\frac{q}{p}}\right) c+S\left(a, \frac{p-q}{p} K^{\frac{q}{p}}\right)\right.} \\
& \left.+\int_{a}^{b} g(\tau) S\left(\tau, \frac{p-r}{p} K^{\frac{r}{p}}\right) \Delta \tau\right] e_{p}(t, a)+\int_{a}^{t} Q(\tau) e_{P}(t, \sigma(\tau)) \Delta \tau, \\
l(t)= & \frac{r}{q} K^{\frac{r-q}{p}} e_{p}(t, a)
\end{aligned}
$$

and

$$
\begin{aligned}
Q(t)= & S^{\Delta}\left(t, \frac{p-q}{p} K^{\frac{q}{p}}\right)+f(\sigma(t), t) S\left(t, \frac{p-q}{p} K^{\frac{q}{p}}\right) \\
& +\int_{a}^{t} f^{\Delta}(t, \tau) S\left(\tau, \frac{p-q}{p} K^{\frac{q}{p}}\right) \Delta \tau, \\
P(t)= & \frac{q}{p} K^{\frac{q-p}{p}} R\left(\sigma(t), \frac{p-q}{p} K^{\frac{q}{p}}\right) h(t)+\frac{R^{\Delta}\left(t, \frac{p-q}{p} K^{\frac{q}{p}}\right)}{R\left(t, \frac{p-q}{p} K^{\frac{q}{p}}\right)} \\
& +f(\sigma(t), t)+\int_{a}^{t} f^{\Delta}(t, \tau) \Delta \tau,
\end{aligned}
$$

with

$$
\int_{a}^{b} \frac{R\left(\tau, \frac{p-r}{p} K^{\frac{r}{p}}\right) g(\tau)}{R\left(\tau, \frac{q-p}{p} K^{\frac{q}{p}}\right)} l(\tau) \Delta \tau<1
$$


Proof. Define a function $z(t)$ as follows

$z(t)=c+\int_{a}^{t} h(s)\left[S\left(s, u^{q}(s)\right)+\int_{a}^{s} f(s, \tau) S\left(\tau, u^{q}(\tau)\right) \Delta \tau+\int_{a}^{b} g(\tau) S\left(\tau, u^{r}(\tau)\right) \Delta \tau\right] \Delta s$

then

$(4.18) z^{\Delta}(t)=h(t)\left[S\left(t, u^{q}(t)\right)+\int_{a}^{t} f(t, \tau) S\left(\tau, u^{q}(\tau)\right) \Delta \tau+\int_{a}^{b} g(\tau) S\left(\tau, u^{r}(\tau)\right) \Delta \tau\right]$.

Using Lemma 2.4 and (4.9), inequality (4.18) becomes

$$
\begin{aligned}
z^{\Delta}(t) \leq & h(t)\left[\frac{q}{p} K^{\frac{q-p}{p}} R\left(t, \frac{p-q}{p} K^{\frac{q}{p}}\right) z(t)+S\left(t, \frac{p-q}{p} K^{\frac{q}{p}}\right)\right. \\
& +\int_{a}^{t} f(t, \tau)\left(\frac{q}{p} K^{\frac{q-p}{p}} R\left(\tau, \frac{p-q}{p} K^{\frac{q}{p}}\right) z(\tau)+S\left(\tau, \frac{p-q}{p} K^{\frac{q}{p}}\right)\right) \Delta \tau \\
& \left.+\int_{a}^{b} g(\tau)\left(\frac{r}{p} K^{\frac{r-p}{p}} R\left(\tau, \frac{p-r}{p} K^{\frac{r}{p}}\right) z(\tau)+S\left(\tau, \frac{p-r}{p} K^{\frac{r}{p}}\right)\right) \Delta \tau\right] .
\end{aligned}
$$

Denote a function $v(t)$ by

$$
\begin{aligned}
v(t)= & \frac{q}{p} K^{\frac{q-p}{p}} R\left(t, \frac{p-q}{p} K^{\frac{q}{p}}\right) z(t)+S\left(t, \frac{p-q}{p} K^{\frac{q}{p}}\right) \\
& +\int_{a}^{t} f(t, \tau)\left(\frac{q}{p} K^{\frac{q-p}{p}} R\left(\tau, \frac{p-q}{p} K^{\frac{q}{p}}\right) z(\tau)+S\left(\tau, \frac{p-q}{p} K^{\frac{q}{p}}\right)\right) \Delta \tau \\
& +\int_{a}^{b} g(\tau)\left(\frac{r}{p} K^{\frac{r-p}{p}} R\left(\tau, \frac{p-r}{p} K^{\frac{r}{p}}\right) z(\tau)+S\left(\tau, \frac{p-r}{p} K^{\frac{r}{p}}\right)\right) \Delta \tau
\end{aligned}
$$

Obviously, $v$ is a delta differentiable non-decreasing function satisfying

$$
\begin{aligned}
v(a)= & \frac{q}{p} K^{\frac{q-p}{p}} R\left(a, \frac{p-q}{p} K^{\frac{q}{p}}\right) c+S\left(a, \frac{p-q}{p} K^{\frac{q}{p}}\right) \\
& +\int_{a}^{b} g(\tau)\left(\frac{r}{p} K^{\frac{r-p}{p}} R\left(\tau, \frac{p-r}{p} K^{\frac{r}{p}}\right) z(\tau)+S\left(\tau, \frac{p-r}{p} K^{\frac{r}{p}}\right)\right) \Delta \tau
\end{aligned}
$$

and

$$
\frac{q}{p} K^{\frac{q-p}{p}} R\left(t, \frac{p-q}{p} K^{\frac{q}{p}}\right) z(t) \leq v(t)
$$


Based on a straightforward computation, one has

$$
\begin{aligned}
v^{\Delta}(t)= & \frac{q}{p} K^{\frac{q-p}{p}} R\left(\sigma(t), \frac{p-q}{p} K^{\frac{q}{p}}\right) z^{\Delta}(t)+\frac{q}{p} K^{\frac{q-p}{p}} R^{\Delta}\left(t, \frac{p-q}{p} K^{\frac{q}{p}}\right) z(t) \\
& +S^{\Delta}\left(t, \frac{p-q}{p} K^{\frac{q}{p}}\right) \\
& +f(\sigma(t), t)\left[\frac{q}{p} K^{\frac{q-p}{p}} R\left(t, \frac{p-q}{p} K^{\frac{q}{p}}\right) z(t)+S\left(t, \frac{p-q}{p} K^{\frac{q}{p}}\right)\right] \\
& +\int_{a}^{t} f^{\Delta}(t, \tau)\left[\frac{q}{p} K^{\frac{q-p}{p}} R\left(\tau, \frac{p-q}{p} K^{\frac{q}{p}}\right) z(\tau)+S\left(\tau, \frac{p-q}{p} K^{\frac{q}{p}}\right)\right] \Delta \tau .
\end{aligned}
$$

It implies that

$$
\begin{aligned}
v^{\Delta}(t) \leq & {\left[\frac{q}{p} K^{\frac{q-p}{p}} R\left(\sigma(t), \frac{p-q}{p} K^{\frac{q}{p}}\right) h(t)+\frac{R^{\Delta}\left(t, \frac{p-q}{p} K^{\frac{q}{p}}\right)}{R\left(t, \frac{p-q}{p} K^{\frac{q}{p}}\right)}+f(\sigma(t), t)+\int_{a}^{t} f^{\Delta}(t, \tau) \Delta \tau\right] } \\
& \times v(t)+S^{\Delta}\left(t, \frac{p-q}{p} K^{\frac{q}{p}}\right)+f(\sigma(t), t) S\left(t, \frac{p-q}{p} K^{\frac{q}{p}}\right) \\
& +\int_{a}^{t} f^{\Delta}(t, \tau) S\left(\tau, \frac{p-q}{p} K^{\frac{q}{p}}\right) \Delta \tau \\
= & P(t) v(t)+Q(t),
\end{aligned}
$$

where $P(t)$ and $Q(t)$ are defined as in (4.17).

Following the same arguments as in the proof of Theorem 3.2, we obtain the desired inequality (4.16).

Corollary 4.1. Assume that all assumptions of Theorem 3.2 hold. Let $c(t)$ be nondecreasing function in $C_{r d}\left([a, b]_{\mathbb{T}}, \mathbb{R}_{+}^{*}\right)$ which satisfies $S^{\Delta}(t, 0) c(t) \geq S(t, 0) c^{\Delta}(t)$ and $R^{\Delta}(t, 0) \geq 0$ on $[\alpha, b]_{\mathbb{T}}^{k}$, where $S$ is defined as in (4.1). Then

$$
\begin{gathered}
u(t) \\
\leq c(t)+\int_{a}^{t} h(s)\left[S(s, u(s))+\int_{a}^{s} f(s, \tau) S(\tau, u(\tau)) \Delta \tau+\int_{a}^{b} g(\tau) S(\tau, u(\tau)) \Delta \tau\right] \Delta s,
\end{gathered}
$$

implies

$$
u(t) \leq c(t)\left\{1+\int_{a}^{t} h(s)\left[m(s)+\frac{l(s) \int_{a}^{b} g(\tau) m(\tau) \Delta \tau}{1-\int_{a}^{b} g(\tau) l(\tau) \Delta \tau}\right] \Delta s\right\}
$$


for $t \in[a, b]_{\mathbb{T}}^{k}$, where

$$
\begin{aligned}
m(t) & =\left[R(a, 0)+M(a, 0)+\int_{a}^{b} g(\tau) M(\tau, 0) \Delta \tau\right] e_{p}(t, a)+\int_{a}^{t} e_{P}(t, \sigma(\tau)) Q(\tau) \Delta \tau, \\
l(t) & =e_{p}(t, a)
\end{aligned}
$$

and

$$
\begin{aligned}
& Q(t)=M^{\Delta}(t, 0)+f(\sigma(t), t) M(t, 0)+\int_{a}^{t} f^{\Delta}(t, \tau) M(\tau, 0) \Delta \tau, \\
& P(t)=R(\sigma(t), 0) h(t)+\frac{R^{\Delta}(t, 0)}{R(t, 0)}+f(\sigma(t), t)+\int_{a}^{t} f^{\Delta}(t, \tau) \Delta \tau,
\end{aligned}
$$

with

$$
\int_{a}^{b} g(\tau) l(\tau) \Delta \tau<1
$$

and

$$
M(t, u(t))=\frac{1}{c(t)} S(t, c(t) u(t)) .
$$

Proof. Since $c(t)$ is non-decreasing, from (4.19) we have

$$
\begin{aligned}
w(t) \leq & +\int_{a}^{t} h(s)\left[\frac{1}{c(s)} S(s, c(s) w(s))+\int_{a}^{s} f(s, \tau) \frac{1}{c(\tau)} S(\tau, c(\tau) w(\tau)) \Delta \tau\right. \\
& \left.+\int_{a}^{b} g(\tau) \frac{1}{c(\tau)} S(\tau, c(\tau) w(\tau)) \Delta \tau\right] \Delta s .
\end{aligned}
$$

Let

$$
M(t, x)=\frac{1}{c(t)} S(t, c(t) x) .
$$

Furthermore, one can easily see that $M(t, x)$ satisfies inequality (4.1), since

$$
0 \leq M(t, x)-M(t, y) \leq R_{1}(t, y)(x-y), \quad x \geq y \geq 0,
$$

with $R_{1}(t, y)=R(t, c(t) y(t))$. Taking into account that $S^{\Delta}(t, 0) c(t) \geq S(t, 0) c^{\Delta}(t)$ and $R^{\Delta}(t, 0) \geq 0$ on $[a, b]_{\mathbb{T}}^{k}$ and a suitable application of Theorem 4.2 for $(p=q=$ $r=c=1$ ), we deduce inequality (4.19).

In this section, further generalizations of the Bellman-Bihari inequality are obtained, where the nonlinear functions appearing on the right side belong to certain classes of functions.

Theorem 4.3. Consider $u, g, h \in C_{r d}\left([a, b]_{\mathbb{T}}, \mathbb{R}_{+}\right)$. Let $n: \mathbb{R}_{+} \rightarrow \mathbb{R}_{+}$a continuous non-decreasing function with $n(u)>0$, for $u>0$ and $N$ is defined as in Lemma 2.3. 
If $f$ is defined as in Lemma 2.2 such that $f(t, s) \geq 0$ and $f^{\Delta}(t, s) \geq 0$ for $t, s \in[a, b]_{\mathbb{T}}$ with $s \leq t$. Then

$$
u(t) \leq c+\int_{a}^{t} h(s)\left[n(u(s))+\int_{a}^{s} f(s, \tau) n(u(\tau)) \Delta \tau+\int_{a}^{s} g(\tau) n(u(\tau)) \Delta \tau\right] \Delta s,
$$

implies

$$
u(t) \leq N^{-1}\left(N(c)+\int_{a}^{t} h(s)\left(1+\int_{a}^{s} f(s, \tau) \Delta \tau+\int_{a}^{s} g(\tau) \Delta \tau\right) \Delta s\right)
$$

for $t \in[a, b]_{\mathbb{T}}^{k}$, with

$$
N(c)+\int_{a}^{t} h(s)\left(1+\int_{a}^{s} f(s, \tau) \Delta \tau+\int_{a}^{s} g(\tau) \Delta \tau\right) \Delta s \in \operatorname{Dom}\left(N^{-1}\right) .
$$

Proof. Let $z(t)$ be the right side of (4.20), then

$$
u(t) \leq z(t)
$$

Using properties of $n$, one can get

$$
z^{\Delta}(t) \leq h(t)\left[n(z(t))+\int_{a}^{t} f(t, \tau) n(z(\tau)) \Delta \tau+\int_{a}^{t} g(\tau) n(z(\tau)) \Delta \tau\right]
$$

then (4.24) can be reformulated as

$$
z^{\Delta}(t) \leq h(t)\left(1+\int_{a}^{t} f(t, \tau) \Delta \tau+\int_{a}^{t} g(\tau) \Delta \tau\right) n(z(t)) .
$$

The inequality (4.25) implies

$$
\frac{z^{\Delta}(t)}{n(z(t))} \leq h(t)\left(1+\int_{a}^{t} f(t, \tau) \Delta \tau+\int_{a}^{t} g(\tau) \Delta \tau\right) .
$$

Integrating both sides of the above inequality from $a$ to $t$ and applying Lemma 2.3, one can obtain

$$
N(z(t)) \leq N(z(a))+\int_{a}^{t} h(s)\left(1+\int_{a}^{s} f(s, \tau) \Delta \tau+\int_{a}^{s} g(\tau) \Delta \tau\right) \Delta s,
$$

then

$$
z(t) \leq N^{-1}\left(N(c)+\int_{a}^{t} h(s)\left(1+\int_{a}^{s} f(s, \tau) \Delta \tau+\int_{a}^{s} g(\tau) \Delta \tau\right) \Delta s\right),
$$

by (4.23), we have the desired inequality (4.21).

Corollary 4.2. If all hypotheses of Theorem 4.3 are satisfied with $n^{-1}(] 0,+\infty[) \subset$ ] $0,+\infty[$, then

$$
n(u(t)) \leq c+\int_{a}^{t} h(s)\left[u(s)+\int_{a}^{s} f(s, \tau) u(\tau) \Delta \tau+\int_{a}^{s} g(\tau) u(\tau) \Delta \tau\right] \Delta s,
$$


implies

(4.27) $u(t) \leq n^{-1}\left(W^{-1}\left(W(c)+\int_{a}^{t} h(s)\left(1+\int_{a}^{s} f(s, \tau) \Delta \tau+\int_{a}^{s} g(\tau) \Delta \tau\right) \Delta s\right)\right)$,

for $t \in[a, b]_{\mathbb{T}}^{k}$, with

$$
W(x)=\int_{x_{0}}^{x} \frac{d s}{n^{-1}(s)}, \quad x>x_{0}>0
$$

and

$$
\begin{array}{r}
\left(W(c)+\int_{a}^{t} h(s)\left(1+\int_{a}^{s} f(s, \tau) \Delta \tau+\int_{a}^{b} g(\tau) \Delta \tau\right) \Delta s\right) \in \operatorname{Dom}\left(W^{-1}\right), \\
W^{-1}\left(W(c)+\int_{a}^{t} h(s)\left(1+\int_{a}^{s} f(s, \tau) \Delta \tau+\int_{a}^{s} g(\tau) \Delta \tau\right) \Delta s\right) \in \operatorname{Dom}\left(n^{-1}\right) .
\end{array}
$$

Proof. Let $z(t)$ be the right side of (4.26). Using properties of $n$, one can get

$$
u(t) \leq n^{-1}(z(t))
$$

then

$z(t) \leq c+\int_{a}^{t} h(s)\left[n^{-1}(z(s))+\int_{a}^{s} f(s, \tau) n^{-1}(z(\tau)) \Delta \tau+\int_{a}^{s} g(\tau) n^{-1}(z(\tau)) \Delta \tau\right] \Delta s$.

Applying Theorem 4.3, one can obtain the desired inequality (4.27).

Corollary 4.3. If all hypotheses of Theorem 4.3 are satisfied, $n$ belongs to class $\mathcal{S}$ (see Definition 2.2), $c(t)$ be non-decreasing function in $C_{r d}\left([a, b]_{\mathbb{T}}^{k}, \mathbb{R}_{+}^{*}\right)$. If

$$
\leq c(t)+\int_{a}^{t} h(s)\left[n(u(s))+\int_{a}^{s} f(s, \tau) n(u(\tau)) \Delta \tau+\int_{a}^{s} g(\tau) n(u(\tau)) \Delta \tau\right] \Delta s,
$$

then

(4.29) $u(t)$

$$
\leq \max (c(t), 1)\left(N^{-1}\left(N(1)+\int_{a}^{t} h(s)\left(1+\int_{a}^{s} f(s, \tau) \Delta \tau+\int_{a}^{s} g(\tau) \Delta \tau\right) \Delta s\right)\right),
$$

for $t \in[a, b]_{\mathbb{T}}^{k}$, with

$$
N(1)+\int_{a}^{t} h(s)\left(1+\int_{a}^{s} f(s, \tau) \Delta \tau+\int_{a}^{s} g(\tau) \Delta \tau\right) \Delta s \in \operatorname{Dom}\left(N^{-1}\right),
$$

Proof. Denote $b(t)=\max (c(t), 1)$. Then $(4.28)$ can be rewritten as

$$
\frac{u(t)}{b(t)} \leq 1+\int_{a}^{t} \frac{h(s)}{b(s)}\left[n(u(s))+\int_{a}^{s} f(s, \tau) n(u(\tau)) \Delta \tau+\int_{a}^{s} g(\tau) n(u(\tau)) \Delta \tau\right] \Delta s .
$$


Let $z(t)=\frac{u(t)}{b(t)}$. Since $n$ belongs to class $\mathcal{S}$, one has

$$
z(t) \leq 1+\int_{a}^{t} h(s)\left[n(z(s))+\int_{a}^{s} f(s, \tau) n(z(\tau)) \Delta \tau+\int_{a}^{s} g(\tau) n(z(\tau)) \Delta \tau\right] \Delta s .
$$

As $n$ is a non-decreasing function, using Theorem 4.3, one can obtain the desired inequality (4.29).

Corollary 4.4. Assume that the hypotheses of Theorem 4.3 hold and $n$ belongs to class $\mathcal{T}$ (see Definition 2.3). Then

$$
n(u(t)) \leq c(t)+\int_{a}^{t} h(s)\left[u(s)+\int_{a}^{s} f(s, \tau) u(\tau) \Delta \tau+\int_{a}^{s} g(\tau) u(\tau) \Delta \tau\right] \Delta s
$$

implies

$$
\begin{aligned}
u(t) \leq & \max (c(t), 1) n^{-1}\left(W ^ { - 1 } \left(W(1)+\int_{a}^{t} h(s)\left(1+\int_{a}^{s} f(s, \tau) \Delta \tau\right.\right.\right. \\
& \left.\left.\left.+\int_{a}^{s} g(\tau) \Delta \tau\right) \Delta s\right)\right)
\end{aligned}
$$

for $t \in[a, b]_{\mathbb{T}}^{k}$, with

$$
\begin{array}{r}
\left(W(1)+\int_{a}^{t} h(s)\left(1+\int_{a}^{s} f(s, \tau) \Delta \tau+\int_{a}^{s} g(\tau) \Delta \tau\right) \Delta s\right) \in \operatorname{Dom}\left(W^{-1}\right), \\
W^{-1}\left(W(1)+\int_{a}^{t} h(s)\left(1+\int_{a}^{s} f(s, \tau) \Delta \tau+\int_{a}^{s} g(\tau) \Delta \tau\right) \Delta s\right) \in \operatorname{Dom}\left(n^{-1}\right) .
\end{array}
$$

Proof. Denote $b(t)=\max (c(t), 1)$. Then, (4.30) can be reformulated as

$$
\frac{n(u(t))}{b(t)} \leq 1+\int_{a}^{t} \frac{h(s)}{b(s)}\left[u(s)+\int_{a}^{s} f(s, \tau) u(\tau) \Delta \tau+\int_{a}^{b} g(\tau) u(\tau) \Delta \tau\right] \Delta s .
$$

Let $z(t)=\frac{u(t)}{b(t)}$. Since $n$ belongs to class $\mathcal{T}$, from (4.32), one has

$$
n(z(t)) \leq 1+\int_{a}^{t} h(s)\left[z(s)+\int_{a}^{s} f(s, \tau) z(\tau) \Delta \tau+\int_{a}^{b} g(\tau) z(\tau) \Delta \tau\right] \Delta s .
$$

Applying Corollary 4.2, we obtain the desired inequality (4.31).

\section{Applications}

In this section we present some examples for our main results to investigate certain properties of solutions of dynamic equation on time scales.

Example 5.1. Consider the following general mixed non-linear integral equation

$$
y^{p}(t)=x(t)+\int_{a}^{t} F\left(s, y^{q}(s)\right) \Delta s+\int_{a}^{b} G\left(s, y^{r}(s)\right) \Delta s,
$$


for $t \in[a, b]_{\mathbb{T}}$, where $p \geq q>0, p \geq r>0, y(t)$ is unknown function, $x \in$ $C_{r d}\left([a, b]_{\mathbb{T}}, \mathbb{R}\right), F, G \in C_{r d}\left([a, b]_{\mathbb{T}} \times \mathbb{R}, \mathbb{R}\right)$. Suppose that the functions $x, y, F, G$ in (5.1) satisfy the following conditions:

$$
\begin{aligned}
|x(t)| & \leq c(t), \\
\left|F\left(s, y^{q}(s)\right)\right| & \leq f(s)|y|^{q}, \\
\left|G\left(s, y^{r}(s)\right)\right| & \leq g(s)|y|^{r},
\end{aligned}
$$

where $c(t), f(t), g(t)$ are in $C_{r d}\left([a, b]_{\mathbb{T}}, \mathbb{R}_{+}\right)$.

Proposition 5.1. Assume that $y(t)$ is the unique solution of equation (5.1) and $\int_{a}^{b} g(s) l(s) \Delta s<1$, then

$$
|y(t)| \leq\left\{m(t)+\frac{l(t) \int_{a}^{b} g(s) m(s) \Delta s}{1-\int_{a}^{b} g(s) l(s) \Delta s}\right\}^{\frac{1}{p}}
$$

where $m(t)$ is defined as in Theorem 3.1 and

$$
l(t)=\frac{r}{p} k^{\frac{r-p}{p}} e_{p}(t, a) .
$$

Proof. From equations (5.1) and (5.2), we obtain

$$
|y(t)|^{p} \leq c(t)+\int_{a}^{t} f(s)|y(s)|^{q} \Delta s+\int_{a}^{b} g(s)|y(s)|^{r} \Delta s .
$$

Now, an application of Theorem 3.1 for $f(t, s)=f(t)$, yields the required estimate in $(5.3)$.

Example 5.2. Consider the following initial value problem

$$
\begin{aligned}
y^{\Delta}(t) & =h(t)\left[n(y(t))+\int_{a}^{t} f(t, s) n(y(s)) \Delta s+\int_{a}^{t} g(s) n(y(s)) \Delta s\right], \\
y(a) & =c
\end{aligned}
$$

where $h(t), f(t, s), g(t)$ and $n(t)$ are as defined in Theorem 4.3, and $c$ is a constant.

Proposition 5.2. Assume that $y(t)$ is the unique solution of (5.4). Then

$$
u(t) \leq N^{-1}\left(N(c)+\int_{a}^{t} h(s)\left(1+\int_{a}^{s} f(s, \tau) \Delta \tau+\int_{a}^{s} g(\tau) \Delta \tau\right) \Delta s\right),
$$

with

$$
\left(N(c)+\int_{a}^{t} h(s)\left(1+\int_{a}^{s} f(s, \tau) \Delta \tau+\int_{a}^{s} g(\tau) \Delta \tau\right) \Delta s\right) \in \operatorname{Dom}\left(N^{-1}\right) .
$$

Proof. If $y(t)$ is the unique solution of (5.4), then $y(t)$ can be expressed as

$$
y(t)=c+\int_{a}^{t} h(s)\left[n(y(s))+\int_{a}^{s} f(s, \tau) n(y(\tau)) \Delta \tau+\int_{a}^{s} g(\tau) n(y(\tau)) \Delta \tau\right] \Delta s .
$$


Then

$$
|y(t)| \leq|c|+\int_{a}^{t} h(s)\left[n(|y(s)|)+\int_{a}^{s} f(s, \tau) n(|y(\tau)|) \Delta \tau+\int_{a}^{s} g(\tau) n(|y(\tau)|) \Delta \tau\right] \Delta s .
$$

Applying Theorem 4.3, we obtain the desired inequality (5.5).

\section{ACKNOWLEDGEMENT}

The authors are very grateful to the referees for their helpful comments and valuable suggestions.

\section{REFERENCES}

[1] B. Aulbach and S. Hilger, A unified approach to continuous and discrete dynamics, Qualitative theory of differential equations (Szeged, 1988), vol. 53 of Colloq. Math. Soc. János Bolyai, North-Holland, Amsterdam, 1990, pp. 37-56.

[2] D. Bainnov and P. Simeonov, Integral Inequalities and Applications, vol. 57 of Mathematics and its Applications (East European Series), Kluwer Academic Publishers Group, Dordrecht, 1992, translated by R. A. M. Hoksbergen and V. Covachev.

[3] B. Ben Nasser, K. Boukerrioua and M. A. Hammami, On the stability of perturbed time scale systems using integral inequalities, Appl. Sci. 16 (2014), 56-71.

[4] B. Ben Nasser, K. Boukerrioua and M. A. Hammami, On stability and stabilization of perturbed time scale systems with Gronwall inequalities, Zh. Mat. Fiz. Anal. Geom. 11 (2015), 207-235.

[5] M. Bohner and A. Peterson, Dynamic Equations on Time Scales, Birkhäuser Basel, Boston, MA, 2001.

[6] M. Bohner and A. Peterson (Eds.), Advances in Dynamic Equations on Time Scales, Birkhäuser Basel, Boston, MA, 2003.

[7] K. Boukerrioua, Note on some nonlinear integral inequalities and applications to differential equations, Int. J. Differ. Equ. (2011), Article ID 456216.

[8] K. Boukerrioua, Note on some nonlinear integral inequalities on time scales and applications to dynamic equations, J. Adv. Res. Appl. Math. 5 (2013), 1-12.

[9] K. Boukerrioua and A. Guezane-Lakoud, Some nonlinear integral inequalities arising in differential equations, Electron. J. Differential Equations 2008 (2008), Article ID 80.

[10] K. Cheng and C. Guo, New explicit bounds on Gamidov type integral inequalities for functions in two variables and their applications, Abstr. Appl. Anal. 2014 (2014), Article ID 539701.

[11] K. Cheng, C. Guo and M. Tang, Some nonlinear Gronwall-Bellman-Gamidov integral inequalities and their weakly singular analogues with applications, Abstr. Appl. Anal. 2014 (2014), Article ID 562691.

[12] S. S. Dragomir, Some Gronwall Type Inequalities and Applications, Nova Science Publishers, Hauppauge, NY, 2003.

[13] R. A. C. Ferreira and D. F. M. Torres, Generalizations of Gronwall-Bihari inequalities on time scales, J. Difference Equ. Appl. 15 (2009), 529-539.

[14] Sh. G. Gamidov, Certain integral inequalities for boundary value problems of differential equations, Differ. Uravn. 5 (1969), 463-472.

[15] F. Jiang and F. Meng, Explicit bounds on some new nonlinear integral inequalities with delay, J. Comput. Appl. Math. 205 (2007), 479-486.

[16] S. D. Kendre and S. G. Latpate, On some mixed integral inequalities and its applications, Theoretical Mathematics and Applications 5 (2015), 1-14.

[17] B. G. Pachpatte, Inequalities for Differential and Integral Equations, vol. 197 of Mathematics in Science and Engineering, Academic Press, Inc., San Diego, CA, 1998. 
[18] B. G. Pachpatte, A note on certain integral inequality, Tamkang J. Math. 33 (2002), 353-358.

[19] B. G. Pachpatte, Explicit bounds on Gamidov type integral inequalities, Tamkang J. Math. 37 (2006), 1-9.

[20] B. G. Pachpatte, Integral and Finite Difference Inequalities and Applications, vol. 205 of NorthHolland Mathematics Studies, Elsevier Science B.V., Amsterdam, 2006.

${ }^{1}$ LANOS LABORATORY

UNIVERSITY OF BADJI-MOKHTAR

Annaba, Algeria

E-mail address: khaledv2004@yahoo.fr

E-mail address: Meziri-Imen91@hotmail.fr

${ }^{2}$ UNIVERSITY OF GUELMA

Guelma, Algeria

E-mail address: tchiheb@yahoo.fr 